\title{
A utilização de protozoários como organismos bioindicadores em ensaios ecotoxicológicos e suas respostas à exposição a fármacos e outros compostos
}

The use of protozoans as bioindicators organism in ecotoxicological tests and their responses when exposed to drugs and other compounds

El uso de protozoos como bioindicadores en pruebas ecotoxicológicas y sus respuestas cuando se exponen a fármacos y otros compuestos

Nelson Giovanini Junior Mestrando em Engenharia Civil, FEIS - UNESP, Brasil nelsongiovanini@outlook.com

Mariana Barbosa de Carvalho Mestranda em Engenharia Civil, FEIS - UNESP, Brasil maa.carvalho@hotmail.com 


\section{RESUMO}

Protozoários são microrganismos eucarióticos geralmente unicelulares e heterotróficos extensamente estudados pela biologia e vastamente difundidos nos diversos ecossistemas aquáticos em todo o mundo. Este trabalho busca fazer uma revisão bibliográfica sobre o uso das mais diversas espécies de protozoários - com um maior enfoque para os protozoários ciliados - como biondicadores em ensaios ecotoxicológicos e toxicológicos. Foram analisados vários estudos que usam os protozoários como organismos testes para avaliar os mais diversos compostos orgânicos e químicos, buscando - brevemente - avaliar os efeitos desses compostos sobre a espécie em estudo. Foram encontrados diversos trabalhos na área, principalmente com a utilização de pesticidas e fármacos, mostrando a influência desses compostos no crescimento e metabolismos dos protozoários. Verificou-se que os protozoários como bioindicadores possuem características que os tornam excelente escolha para os ensaios ecotoxicológicos quando comparados à outras espécies, afirmando as suas propriedades como organismos teste

PALAVRAS-CHAVE: protozoários, ecotoxicologia, bioindicadores.

\section{ABSTRACT}

Protozoans are microorganisms eukaryotic normally unicellular and heterotrophies widely studied in biology and vastly widespread in many aquatic ecosystems around the world. This work pursuit to make a bibliographical study about the use of many protozoan species - with a bigger focus on ciliated protozoans - as bioindicators in ecotoxicological and toxicological tests. Were analyzed several studies that used protozoans as tests organism to evaluate many organic and chemical compounds, looking for - briefly - evaluate the effect of those compounds over the specie being studied. Were found many papers in this field of study, mostly utilizing pesticides and pharmaceuticals, showing the influence of these compounds in the protozoans' metabolism and growth. It was also noted that protozoans as bioindicators have features that make them an excellent choice for ecotoxicological essays when compared to other species, affirming their properties as tests organism.

KEYWORDS: protozoans, ecotoxicology, bioindicators.

\section{RESUMEN}

Los protozoos son microorganismos eucariotas normalmente unicelulares y heterotróficos ampliamente estudiados en biología y muy extendidos en muchos ecosistemas acuáticos de todo el mundo. Este trabajo busca realizar un estudio bibliográfico sobre el uso de muchas especies de protozoarios -con un mayor enfoque en los protozoarios ciliados- como bioindicadores en las pruebas ecotoxicológicas y toxicológicas. Se analizaron varios estudios que utilizaron protozoos como organismo de pruebas para evaluar muchos compuestos orgánicos y químicos, buscando - brevemente - evaluar el efecto de esos compuestos sobre la especie que se está estudiando. Se encontraron muchos trabajos en este campo de estudio, en su mayoría utilizando pesticidas y productos farmacéuticos, mostrando la influencia de estos compuestos en el metabolismo y crecimiento de los protozoarios. También se observó que los protozoos como bioindicadores tienen características que los hacen una excelente opción para ensayos ecotoxicológicos en comparación con otras especies, afirmando sus propiedades como organismo de pruebas.

PALABRAS CLAVE: protozoos, ecotoxicología, bioindicadores 


\section{1 - INTRODUÇÃO E CONTEXTUALIZAÇÃO DO ASSUNTO}

Ecotoxicologia é a ciência que integra conhecimentos da ecologia e toxicologia de modo a avaliar e prever os efeitos de contaminantes nos sistemas biológicos, sendo a Ecotoxicologia Aquática um ramo que estuda esses efeitos no ecossistema aquático (KRULL, 2010, apud MOISEENKO, 2008).

Os campos da ecologia e toxicologia desenvolveram-se como disciplinas separadas durante o século passado com jornais exclusivos, ferramentas únicas e jargões distintos reforçando a ideia de separação entre as duas. Enquanto os ecologistas focavam em estudar os fatores bióticos e abióticos da distribuição das espécies e suas interações, toxicologistas tradicionalmente focavam em testes toxicológicos em espécies individuais. Existe, entretanto, o campo de estudo da ecotoxicologia - um nome que naturalmente implica a junção das ideias da ecologia e toxicologia (RELYEA, 2006).

A ascensão da ecotoxicologia como um campo de estudo (também nomeado como "toxicologia ambiental") começou a ter mais importância a partir da década de 60 com a primeira definição formal vindo de Tuhaut (1977) que considerou a ecotoxicologia como sendo um ramo da toxicologia preocupada com os efeitos dos poluentes na constituição de um ecossistema integrado (RELYEA, 2006). Desde a definição dada por Tuhaut, pouco mudou com referência a ela, entretanto diversos estudos foram publicados buscando entender e solucionar problemas ambientais com base em ensaios utilizando possíveis contaminantes analisando a resposta de diversas espécies aquáticas quando expostas a esses compostos.

\section{1 - Breve histórico da análise e compostos estudados}

Um dos primeiros compostos químicos a ser analisado - e também um grande responsável pelo crescimento da ecotoxicologia como um campo de estudo - foi o inseticida DDT (dichlordipheny/trichlorethane) utilizado em larga escala entre os anos de 1940 e 1950 para o controle de pragas na agricultura (WERNER et al., 2012). Sendo assim, desde a década de 60, leis ambientais entraram em vigor em diversas nações juntamente com diversos estudos e pesquisas voltados para a análise de compostos tóxicos e seus efeitos ambientais quanto ao uso indiscriminado.

Dentro do grande grupo dos pesticidas sintéticos é encontrada a subdivisão dos inseticidas orgânicos sintéticos que tem como principal característica atuar diretamente no sistema nervoso dos animais, sendo assim considerada como a categoria mais tóxica dentro desse grupo. Os inseticidas DDT tem como principal característica biológica acumularem-se no organismo, devido a sua estabilidade e solubilidade em gordura, sendo um composto que aumenta juntamente com a cadeia trófica, logo, encontram-se concentrações maiores em organismos no topo da cadeia alimentar (WERNER et al., 2012). Diversos - e antigos - estudos já mostraram os efeitos negativos do DDT em múltiplas espécies presentes nos mais variados ecossistemas, como é o caso conhecido e vastamente estudado da ação deste tipo de 
inseticida sobre a formação da casca de ovos de répteis e aves, onde ocorre a diminuição da espessura da casca (HICKEY et al., 1968).

Atualmente, os pesticidas são extremamente necessários para aumentar a produtividade agrícola, entretanto, o maior problema está no uso indiscriminado destes compostos que podem levar a situações ameaçadoras para espécies de determinado ecossistema, como notificado por HICKEY, 1968. Além disso, esses compostos não são totalmente seletivos, uma vez que eles podem causar efeitos tóxicos em organismos diferentes do que o pesticida deveria primeiramente combater, sendo uma ameaça para outros organismos terrestres e aquáticos. E ainda, quando aplicado no solo ou plantas - e dependendo da concentração e frequência de aplicação - os pesticidas estão sujeitos a uma série de processos abióticos e bióticos que podem envolver o transporte desse composto para diferentes matrizes ambientais como solos e corpos de água (MANSANO et al., 2015). Sendo assim, é evidente a facilidade de contaminação de outros ambientes e possivelmente outras espécies, principalmente devido a mobilidade do composto.

A ecotoxicologia iniciou com a avaliação de pesticidas, entretanto, atualmente, a maior preocupação é com os micropoluentes presentes em efluentes doméstico, industriais e rurais. Entre esses micropoluentes encontra-se a classe dos fármacos que engloba os compostos eliminados tanto pelo usuário final doméstico quanto pelo excedente industrial do processo de fabricação. Até recentemente, acreditava-se que os remédios eram metabolizados de certa forma no organismo que seriam excretadas em forma muito diluída sendo ainda misturada com o esgoto e águas de tratamento, tendo como resultado uma concentração ínfima e não impondo riscos aos organismos. Entretanto, essa visão foi abandonada uma vez que técnicas mais avançadas de avaliação detectaram altas doses de fármacos em diferentes ambientes como efluentes de esgotos, águas superficiais e águas subterrâneas (SEILER, 2002). Sendo assim, fica evidente a necessidade de estudar os fármacos como um composto tóxico relevante.

A importância da ecotoxicologia está justamente em avaliar a toxicidade de diversos compostos - começando com os DDT há mais de 50 anos - mensurando seu efeito negativo sobre determinado ambiente. $\mathrm{O}$ uso de organismos vivos como indicadores de perigos ou problemas data antes mesmo das diversas regulações ambientais que hoje estão em vigor, onde ferramentas biológicas baseadas em espécies de animais vivos já eram usadas para detectar anomalias e perigos no ambiente, por exemplo, o uso de pássaros em minas de carvão para avisar aos minerados da presença de níveis elevados de monóxido de carbono e metano (WERNER et al., 2012). Esses organismos vivos são chamados de bioindicadores que segundo AMÉRICO et al., 2017, apud WASHINGTON, 1994, são espécies escolhidas por sua sensibilidade ou tolerância a vários parâmetros, como poluição orgânica ou outros tipos de poluentes.

Com relação ao uso de organismos vivos para o teste de toxicidade de compostos químicos ou orgânicos, existem diversas normas e ensaios que padronizam os experimentos. Esses processos regulamentam os sistemas de testes buscando mensurar a resposta dos organismos representativos de certo ecossistema quanto à vulnerabilidade deles a determinado composto. Os testes são realizados, normalmente, de duas formas: de forma crônica, onde é 
analisado o efeito do composto ao longo de determinando período avaliando a resposta do espécime quanto a diversos fatores - como crescimento, resposta genética, mudanças comportamentais e sistema reprodutivo - e de forma aguda onde é verificada a letalidade do composto em estudo.

\section{2 - Objetivos}

Entre os diversos protistas que podem ser usados como bioindicadores este estudo irá dar enfoque a alguns protozoários ciliados, tendo como principal objetivo analisar a ecotoxicidade de fármacos e outros compostos químicos ou orgânicos.

Esses organismos são extensamente conhecidos e estudados pela biologia sendo classificados como microrganismos eucarióticos geralmente unicelulares e heterotróficos. Segundo AMÉRICO et al., 2017, os protistas são ideais para estudos de ecotoxicidade, pois eles são muito sensíveis a mudanças ambientais alertando precocemente sobre a deterioração de ambientes aquáticos.

Sendo assim, durante toda a extensão do trabalho, os principais tópicos e assuntos a serem considerados são: (1) comportamento dos protozoários como biondicadores; (2) situação dos protozoários frente aos outros organismos testes utilizados em ensaios ecotoxicológicos; (3) respostas dos protozoários quando expostos aos mais diversos tipos de compostos orgânicos ou químicos e (4) breve avaliação de estudos científicos sobre este assunto.

\section{2 - OS PROTOZOÁRIOS COMO BIOINDICADORES}

Espécies indicativas - conforme citado anteriormente chamadas de bioindicadores - são aquelas sensíveis a diversas toxinas funcionando como um indicador das condições de operação de um ecossistema fornecendo uma possível mensuração da qualidade ambiental desse local. Entre essas espécies estão os protozoários ciliados que possuem grande importância nos ambientes aquáticos de água doce, uma vez que eles participam diretamente dos processos de decomposição e alimentação de bactérias. Além disso, eles possuem como característica (PAPADIMITRIOU et al., 2013) responder rapidamente à presença de contaminantes, devido a sua rápida reprodução, sensibilidade e variabilidade dos nichos em que ocupa na cadeia.

Os protistas ciliados frequentemente utilizados para estudos ecotoxicológicos são os Tetrahymena pyriforms (SAUVANT et al., 1999), Colpidium e Paramecium. Alguma de suas características biológicas são (AMÉRICO et al., 2017):

a. Maioria, não todos, unicelulares;

b. Possuem grande superfície relativa com alta taxa metabólica com diversas formas de alimentação sendo eficientes na absorção e liberação de nutrientes;

c. Grande importância no nível trófico mediando o fluxo de substancia e ocupando diversos nichos;

d. Ampla distribuição geográfica com milhares de espécies;

e. Reprodução assexuada e sexuada; 
f. São considerados os organismos unicelulares mais complexos;

g. Chegam a contribuir com $60 \%$ da biomassa total de zooplânctons e

h. Largamente utilizados em ensaios ecotoxicológicos.

Sabe-se que as espécies integrantes dos grupos Tetrahymena, Colpidium e Paramecium são organismos não patogênicos, de vida livre e vastamente distribuídos nos mais diversos ecossistemas. Esta abundância é muito utilizada como uma indicativa da saúde do ambiente aquático em que essas espécies vivem (A. GERHARDT et al., 2010, apud COOLEY et al. 1999). Sendo assim, A. GERHARDT et al., 2010, reforça que o uso dessas espécies pode ajudar a reduzir o número de animais utilizados em testes de toxicidade devido a características biológicas únicas que permitem que eles sejam usados como bioindicadores sensíveis e fáceis de manipular a níveis bioquímicos e populacionais como uma importante parte de um microcosmo simulando uma cadeia alimentar microbiana para avaliar os efeitos diretos e indiretos dos efeitos tóxicos de compostos a um nível de comunidade.

Portanto, comunidades de protozoários podem fornecer informações valiosas na saúde de um ecossistema uma vez que:

a. Protozoários são organismos facilmente comparáveis, largamente distribuídos fazendo-os mais aplicáveis a estudos e;

b. Protozoários são conhecidos por possuem gerações curtas - com altas taxas de reprodução e metabolismo - reagindo rapidamente às mudanças ambientais no ambiente aquáticos.

Sendo assim, JIAN et al., 2005, conclui que os protozoários são melhores indicadores da qualidade da água para mudanças bruscas e para mudanças contínuas ao longo de certo período.

\section{3 - ESTUdOS ECOTOXICOLÓGICOS ENVOLVENDO FÁRMACOS, OUTROS COMPOSTOS E PROTOZOÁRIOS}

Exposto a importância de utilizar os protozoários como bioindicadores, será citado adiante alguns trabalhos de relevância sobre o tema onde diversos compostos tóxicos são utilizados em testes com protozoários buscando entender a interação entre eles determinando e quantificando o nível de toxicidade para a espécie estudada, além disso será analisado alguns trabalhos onde os protozoários são utilizados como indicadores de qualidade do ambiente. Os trabalhos a serem comentados foram escolhidos aleatoriamente em fontes de pesquisa de qualidade sem preferência, buscando atender ao tema pesquisado nesse trabalho. Importante notar que alguns compostos são especificamente fármacos enquanto outros são compostos utilizados pela indústria farmacêutica na produção de fármacos.

\section{1 - Protozoários como indicadores de qualidade de água}

O estudo de MADONI, 2005, avaliou o comportamento de diversas espécies de protozoário ciliados ao longo do curso do rio Pó no noroeste da Itália. Nesse trabalho foram coletadas 
amostras de água e sedimentos em 13 diferentes pontos sendo feito uma análise biológica e estatística das espécies encontradas em cada ponto para determinar suas similaridades e diferenças. Foi concluído que as amostras coletadas nos locais não poluídos foram as mais consistentes e possuem a maior similaridade entre si, enquanto as amostras coletadas em pontos com conhecido depósito de efluentes foram as menos consistentes com pouca similaridade entres as espécies encontradas nessas amostras.

No estudo de JIANG, 2012, foram coletadas amostras de águas na Baia de Jiaozhou no noroeste da China entre junho de 2007 e maio de 2008. Foram coletadas amostras em cinco locais diferentes escolhidos propositalmente uma vez que eram conhecidos impactos ambientais e predominância de ação antropogênica nesses locais. Foram realizados diversos testes biológicos, físicos e estatísticos das amostras coletadas sendo possível concluir que (1) as comunidades de protozoários possuíam significativa diferença de um local para outro; (2) as espécies estavam diretamente ligadas com a quantidade de alguns compostos na água, especificamente o nitrato de nitrogênio e fósforo solúvel, ambos provenientes de depósitos de efluentes; (3) e a diversidade das espécies correlacionam diretamente com o tipo de nutriente encontrado em cada ponto, indicando que a qualidade da água influencia no comportamento dos diferentes tipos de protozoários.

Em outro trabalho, o pesquisador EL-SEREHY et al., 2014, estudou a importância dos ciliados como bioindicadores e um novo tratamento de efluentes utilizando um sistema hidropônico com uma manta de cascalho. As espécies foram divididas em grupos - mais e menos sensíveis a compostos orgânicos e químicos presentes na água - sendo avaliado por meio de análises o antes e depois da passagem pelo sistema de tratamento verificando a predominância das espécies de ciliados em cada caso. Os autores concluíram que o sistema foi eficiente uma vez que a quantidade de ciliados que possuem uma demanda maior de oxigênio foram encontrados em grande quantidade após a passagem pelo sistema de tratamento, indicando uma boa saúde da água e uma eficiência do sistema.

Finalizando, no estudo de CHEN et al., 2008, foi estudada a eficácia de um sistema de tratamento de esgoto recém instalado no sudoeste da China. Foram feitas coletas de amostras de sedimentos antes e depois da água sofrer tratamento utilizando-se o método de análise semelhante ao estudo de EL-SEREHY et al., 2014, onde são feitas divisões na classe de ciliados buscando separá-los conforme o seu comportamento em um ambiente mais ou menos poluído. Com isso, foi possível verificar que antes do tratamento a predominância era apenas de ciliados provenientes de ambientes mais hostis com alta carga de efluentes e após o tratamento foram encontradas diferentes espécies de protozoários ciliados mais sensíveis a poluentes indicando que o sistema foi eficaz em tratar o esgoto.

\section{2 - Ensaios ecotoxocológicos com diversos compostos em protozoários}

Conforme citado, além do uso dos protozoários em avaliações físicas do meio aquático, eles também possuem diversas vantagens como bioindicadores para o estudo toxicológico de compostos químicos e orgânicos. A facilidade de cultivo, o ciclo de vida curto, a falta de estágios complexos de desenvolvimento e o fato de membrana externa dos protozoários 
estarem em contato direto com o meio externo tornam esse grupo susceptível aos ensaios (XU et al., 2004). Na maioria dos testes ecotoxicológicos os resultados são expressos a partir das notações $\mathrm{CL}_{50}, \mathrm{CE}_{50}$ e $\mathrm{Cl}_{50}$, significando:

$\mathrm{CL}_{50}$ : concentração letal para $50 \%$ da população em $\mathrm{mg} / \mathrm{L}$ indicando a mortalidade do organismo testado;

$\mathrm{CE}_{50}$ : concentração de uma substância que causa imobilidade em $50 \%$ dos organismos do ensaio também expressa em $\mathrm{mg} / \mathrm{L}$ e;

$\mathrm{Cl}_{50}$ : concentração de inibição do crescimento em $50 \%$ dos espécimes testados, também expressa em $\mathrm{mg} / \mathrm{L}$.

No estudo conduzido por XU et al., 2004, foi utilizado o composto químico amônia - que é um poluente comum intensamente utilizado pela indústria farmacêutica e na aquicultura encontrado em diversos corpos de água na sua forma ionizada ou não - devido às suas características tóxicas uma vez que passam pelas membranas celulares prejudicando o metabolismo da mesma. Segundo revisão literária feita por XU et al., 2004, a amônia é vastamente encontrada em diversos ambientes aquáticos, assim como os protozoários ciliados, dessa forma o estudo deles focou na análise e efeitos tóxicos da amônia sobre esse tipo de organismo vivo aquático, especificamente a espécie Euplotes vannus. Foram conduzidos ensaios de toxicidade agudo e crônico seguindo padrões adotados pelos pesquisadores. Como resultado eles encontraram que a espécie $E$. vannus consegue sobreviver e reproduzir livremente a concentrações de amônia de até $100 \mathrm{mg} / \mathrm{L}$ sendo a concentração letal $\left(\mathrm{LC}_{50}\right)$ de $7870,46 \mathrm{mg} / \mathrm{L}$, com relação ao ensaio crônico determinou-se que a amônia influenciou em mudanças morfológicas na espécie estudada sendo a principal mudança a níveis intracelulares. Os autores ainda notaram que os resultados indicaram que essa espécie de ciliado foi consideravelmente mais resistente para amônia que uma espécie de larva de ostra, indicando que os protozoários possuem uma grande importância em ambientes aquáticos tóxicos uma vez que atuam na cadeia trófica por mais tempo.

$\mathrm{Na}$ avaliação feita por MIRANDA et al., 2013, foram analisadas a toxicidade de dezessete metais sob a forma de íons de cloreto e o nitrato de prata utilizando o protozoário ciliado da espécie Paramecium caudatum. Os espécimes foram coletados em São Carlos - SP e cultivados sob padrões previamente estabelecidas. As concentrações de testes utilizadas no ensaio foram duas vezes maiores que as adotadas na resolução CONAMA (Conselho Nacional de Meio Ambiente) número 357 onde estipula a concentração máxima permitida de cada um dos metais utilizados no ensaio para poder considerar uma determinada amostra de água potável. Pode-se verificar que a ordem decrescente de toxicidade dos metais em relação à tolerância do protozoário é a seguinte $\mathrm{Hg}>\mathrm{Cu}>\mathrm{Cd}>\mathrm{As}>\mathrm{Ba}>\mathrm{Al}>\mathrm{Ag}>\mathrm{Co}>\mathrm{Ni}>\mathrm{Fe}>\mathrm{Mn}>\mathrm{Sn}>\mathrm{Pb}>\mathrm{Zn}>$ $\mathrm{Cr}>\mathrm{Sr}>\mathrm{Mo}>\mathrm{Li}$. Como resultado os autores concluíram que a espécie estudada possui alta sensibilidade a todos os metais testados, sendo o protozoário um bom indicador dos efeitos tóxicos de metais na agua, uma vez que pode demonstrar o efeito real desses metas em outra comunidades vivas do mesmo ambiente aquático.

Em outro trabalho conduzido por MANSANO et al., 2015, foram estudados os efeitos tóxicos de dois famosos pesticidas - diuron e carforuran - utilizando a espécie de protozoário ciliado Paramecium caudatum. Foram feitos testes toxicológicos agudos e crônicos utilizando a forma 
pura e comercial dos pesticidas sendo encontrado que as formas pura e comercial do diuron possuíam toxicidade semelhante enquanto que a formulação comercial do carbofuran era mais tóxica que sua forma pura. Os resultados dos testes agudos indicaram que as formas comercias e puras do carbofuran são menos tóxicas que o diuron para o teste conduzido (diuron possui $\mathrm{LC}_{50}$ de $62,4 \mathrm{mg} / \mathrm{L}$ para sua forma comercial e o carbofuran de $70,40 \mathrm{mg} / \mathrm{L}$ ). Para os testes crônicos determinou-se que ambos os pesticidas influenciam na reprodução e na formação das populações desses protozoários indicando que esses químicos apresentam um risco biológico para os corpos aquáticos e os organismos que o habitam.

$\mathrm{Na}$ tese defendida por FONSECA, 2006, foi utilizada a espécie de protozoário ciliada Tetrahymena pyriformis como bioindicador no estudo de respostas fisiológicas e bioquímicas à presença de oito corantes utilizados na indústria têxtil. Para isso, foram realizados diversos ensaios toxicológicos em pequena escala analisando o crescimento e morfometria dos espécimes utilizando soluções em diferentes concentrações $(5,25,50$ e 100 ppm) de 8 diferentes corantes anotando a resposta do espécime estudado quanto a respostas para as diferentes concentrações avaliadas. Pretendeu-se, portanto, estudar se os corantes são tóxicos e a que concentração eles produzem tal efeito. Como resultado a autora verificou que não houve sensibilidade tóxica por parte do protozoário estudado para os corantes e suas concentrações avaliadas, indicando que houve apenas algumas mudanças morfométricas como o aumento de tamanho - em alguns indivíduos. Uma sugestão dada pela autora é o de se realizar ensaios com uma mistura desses corantes, e não individualmente, simulando o que acontece em um ambiente aquático.

No trabalho de SEKKAT et al., 1991, foram conduzidos ensaios ecotoxicológicos utilizando cobre, cádmio e o fungicida ferbam com o protozoário Colpidium campylum como bioindicador. O cobre é mundialmente conhecido como um tóxico largamente utilizado na agricultura como fungicida; o cádmio é um resíduo dos fertilizantes a base de fósforo e o ferbam é um tipo de fungicida comercial utilizado em diversas partes do mundo na agricultura e presente em diversos corpos de água. Estudos anteriores indicaram que a mistura entre o cobre e ferbam e o cobre e cádmio possuem efeitos sinergéticos. Portanto, nesse estudo foi avaliada uma mistura dos três compostos considerando a possível sinergia entre eles e também o fato de que nos ambientes aquáticos eles serem encontrados juntos. Os autores concluíram que todos os compostos analisados - individualmente - possuem efeitos tóxicos sobre a espécie estudada para as doses testadas e também demonstraram que mesmo em doses menores a mistura de cádmio-ferbam já era tóxica para o protozoário, confirmando a sinergia entre esses dois compostos e a potencialização da toxicidade.

Outro exemplo de trabalho foi o de WANICK et al., 2008, onde foi determinada a toxicidade aguda do cádmio $\mathrm{Cd}^{+2}$ utilizando o protozoário ciliado Paramecium bursaria por meio de um ensaio ecotoxicológico onde o espécime foi exposto a diferentes concentrações do metal por um período de 24 hora caracterizando o ensaio de forma aguda. Os autores fizeram a avaliação do efeito letal contando o número de organismos mortos sendo a determinação do $\mathrm{CL}_{50}$ utilizando métodos estatísticos. Ficou determinado o valor da concentração letal em 0,64 ppm sendo considerado um valor baixo quando comparado a outras espécies de ciliados, 
indicando o grande potencial do ciliado Paramecium bursaria para ensaios de toxicidade aguda.

Finalizando as análises de estudos tóxicos com os mais diversos compostos utilizando como bioindicadores os protozoários tem-se o trabalho de AMANCHI, 2011, onde foi avaliada a toxicidade do inseticida monocrotophos utilizando o Paramecium caudatum. Foram estudados parâmetros morfológicas do espécime como a viabilidade celular, estrutural e comportamental, e testes nucleicos foram conduzidos utilizando diferentes concentrações do composto estudado. Determinou-se a concentração letal em 332,284 ppm com ênfase na resposta rápida do $P$. caudatum ao composto uma vez que entre 20 a 30 minutos já era possível ver células necrosadas com características típicas de escurecimento do citoplasma, sangramento, vazamento de material interno e mudanças macro nuclear levando a ruptura das células, sendo assim, concluiu-se que este inseticida possui a capacidade de necrosar rapidamente os tecidos da espécie estudada, sugerindo a alta toxicidade dos inseticidas em ambientes aquáticos.

\section{3 - Ensaios ecotoxicológicos em protozoários com fármacos e seus constituintes}

Entre os diversos compostos orgânicos que podem influenciar negativamente nos ecossistemas terrestres estão os compostos farmacêuticos, que devido ao seu crescimento e uso descontrolado, tem se tornado um novo problema ambiental. Devido às suas características químicas e fármaco cinéticas (relacionados com a meia-vida, excreção urinária e/ou fecal e metabolismo do fármaco) a presença desses compostos e seus metabólicos foram detectados em efluentes e estações de tratamento em todo o mundo - que é a maior forma de descarga desses compostos no ambiente (FERRARI et al., 2003). E ainda, os métodos simples de tratamento não são eficientes na remoção de fármacos, sendo assim boa parte desse produto volta para a rede de distribuição, mesmo que em pequena dose, portanto o estudo dos efeitos tóxicos de produtos farmacêuticos é de grande importância.

No estudo realizado por BONNET, 2003, foram analisados 3 fármacos (Cyclosporin-A, Cisplatin e doxorubicin) largamente utilizados no tratamento de câncer, especificamente na quimioterapia, e seus efeitos tóxicos sobre o protozoário ciliado Tetrahymena pyriformis. Foram avaliados os efeitos desses compostos no crescimento da população e nas mudanças morfológicas do organismo estudado. Os resultados mostraram que os 3 compostos eram tóxicos para o Tetrahymena pyriformis com o $\mathrm{LC}_{50}$ em 42.03, 124.37 e $74.62 \mu \mathrm{M}$ para Cyclosporin-A, Cisplatin e doxorubicin respectivamente. Com relação aos efeitos morfológicos, verificou-se que os fármacos causaram o aparecimento de vacúolos digestivo contendo esses elementos, sendo mais presente em altas concentrações. Também foi observada a formação de células deformadas pelas Cisplatin.

Em outro trabalho, os pesquisadores LONAPPAN et al., 2016, avaliaram os efeitos toxicológicos do diclofenac (DCF) - que é uma droga anti-inflamatória usada vastamente em todo o mundo. Estudos anteriores mostraram que - especificamente nas últimas décadas - este fármaco foi detectado nos mais diversos ecossistemas. Devido a essa presença global em ambientes aquáticos os estudos da toxicidade desse fármaco em organismos aquáticos (incluindo os 
protozoários) é de extrema importância. No trabalho os autores mencionam que aproximadamente $50 \%$ do diclofenaco é retirado da água através do tratamento comum de esgoto, sendo que o restante irá para os corpos de água podendo interagir com outros compostos potencializando a sua ação tóxica. Concluiu-se que em pequenas concentrações o composto estudado não tem efeito toxico agudo sobre os organismos aquáticos, entretanto estudos mostraram que o efeito crônico devido a exposição por um período prolongado causa efeitos irreversíveis em diversos organismos.

No trabalho conduzido por KERMICHE et al., 2016, foram analisados dois fármacos não esteroides anti-inflamatórios - Ibuprofen e Diclofenac - quanto aos efeitos toxicológicos sobre a espécie de protozoário Paramecium sp, pesquisando os efeitos destes compostos sobre a taxa de crescimento celular e o metabolismo da respiração. Como resultado os autores encontraram que em baixas concentrações de Ibuprofen (entre 1 e $10 \mu \mathrm{g} / \mathrm{L}$ ) não ocorreu significativa mudanças no crescimento celular e no metabolismo da respiração. Passando esse limiar foi percebida significativa inibição da ocorrência desses dois parâmetros. Percebeu-se também que a espécie estudada foi mais sensível ao Diclofenac com ocorrência de inibição do crescimento celular e inibição da respiração para diferentes concentrações. Finalizando, os autores sugerem uma avaliação do efeito do Ibuprofen em doses baixas uma vez que foi percebido um padrão de comportamento no crescimento celular que pode indicar um efeito de resposta por parte da espécie em estudo.

No trabalho do pesquisador NILSSON, 1989, foi estudado a espécie Tetrahymena e seu comportamento ecotoxicológico quando exposto a diversos tipos de metais ( $\mathrm{Cu}, \mathrm{Zn}, \mathrm{Ni}, \mathrm{Cd}, \mathrm{Hg}$, $\mathrm{Pb}$ ) e alguns tipos de fármacos (chloramphenicol, methotrexate, cisplatin, chloroquine). Foram feitas algumas observações pelo autor quanto ao efeito desses compostos na alteração do PH, que pode influenciar nos resultados. Como resultado observou-se que tanto os fármacos quantos os metais foram responsáveis por alterações nas condições de crescimento da cultura. Na revisão feita por SAUVANT et al., 1999, foi feita uma revisão literária especificamente do protozoário Tetrahymena pyriformis - que é considerado pelo autor como a espécie mais utilizada em estudos científicos conduzidos em protozoários - e sua interação ecotoxicológica com diversos fármacos. As substâncias mais utilizadas são das classes dos antibióticos, antimitóticos, anestésicos e substâncias para tratamento de desordens neurológicas. A maioria dos estudos avaliam o crescimento celular da espécie, sendo que esses compostos têm como característica - a certa concentração - efeitos inibitórios de crescimento e alterações morfológicas. Além disso o autor cita estudos recentes - para a época - de que antibióticos podem inibir a fagocitose do Tetrahymena pyriformis.

\section{4 - CONCLUSÃO}

Este trabalho de revisão teve como intuito mostrar a importância do uso de diversas espécies de protozoários como bioindicadores para a realização de ensaios ecotoxicológicos ou toxicológicos. A consideração desses organismos é feita principalmente devida às suas características previamente citadas - como o rápido crescimento e facilidade de cultivo - que 
permitem uma performance alta se comprada a outras espécies testes como as algas, Daphnia, peixes entre outros.

Também é importante notar que o grande número de trabalhos citados neste texto é suficiente para enfatizar a grande capacidade dos protozoários em funcionar como bioindicadores para estudos da ecotoxicologia seja no campo ambiental ou farmacêutico, analisando o comportamento deles quando exposto à substância testada.

Sendo assim, exposta a relação dos protozoários como bioindicadores, diversos estudos defendem a importância e a necessidade em se padronizar as condições de teste para esses organismos buscando torná-los uma referência de estudo.

\section{REFERÊNCIAS}

AMANCHI N. R., 2011, Acute Toxicity and Cytogenetic Effects of Monocrotophos in Paramecium Caudatum and Oxytricha Fallax. Indian Journal of Fundamental and Applied Life Sciences ISSN: 22316345 (Online). 2011 Vol. 1 (3) July-September, pp.65-70.

AMÉRICO, J. H. P. et al., 2017, Protistas ciliados e seu potencial uso como bioindicadores de qualidade de água. ANAP Brasil, v. 10, n. 18, ISSN 1984-3240.

ANKLEY, G. T. et al. 2006. Toxicogenomics in regulatory ecotoxicology. Environmental Science and Technology 40/13: 4055- 4065.

BONNET J., DUSSER M., BOHATIER J., LAFFOSSE J., 2003, Cytotoxicity assessment of three therapeutic agents, cyclosporin-A, cisplatin and doxorubicin, with the ciliated protozoan Tetrahymena pyriformis. Research in Microbiology 154 (2003) 375-385. doi:10.1016/S0923-2508(03)00085-8

CHEN Qing-Hua, XU Run-Lin, TAM N. F.Y., CHEUNG S. G., SHIN P. K. S., 2008, Use of ciliates (Protozoa: Ciliophora) as bioindicator to assess sediment quality of two constructed mangrove sewage treatment belts in Southern China. Marine Pollution Bulletin 57 (2008) 689-694. doi:10.1016/j.marpolbul.2008.03.015

COOLEY N. R., KELTNER Jr J. M., FORESTER J. (1972) Mirex and Aroclor 1254: effect on and accumulation by Tetrahymena pyriformis strain W. J. Protozool. 19: 636-638

EL-SEREHY H. A., BAHGAT M. M., AL-RASHEID K., AL-MISNED F., MORTUZA G., SHAFIK H., 2013, Cilioprotists as biological indicators for estimating the efficiency of using Gravel Bed Hydroponics System in domestic wastewater treatment. Saudi Journal of Biological Sciences (2014) 21, 250-255. 1319-562X a 2014 Production and hosting by Elsevier B.V. on behalf of King Saud University. http://dx.doi.org/10.1016/j.sjbs.2013.11.003

FERRARI B., PAXEUS N., LO GIUDICE R., POLLIO A., GARRICA J., 2003, Ecotoxicological impact of pharmaceuticals found in treated wastewaters: study of carbamazepine, clofibric acid, and diclofenac. Ecotoxicology and Environmental Safety 55 (2003) 359-370. PII: S 0147-6513(02)00082-9

FONSECA H. A. A., 2006, Estudo in vitro da toxicidade de corantes têxteis azo em Tetrahymena pyriformis. Tese de mestrado. Departamento de Zoologia/ Antropologia Faculdade de Ciências da Universidade do Porto. Outubro de 2006.

GERHARDT, A.; UD-DAULA, A.; SCHRAMM, K. W. Tetrahymena spp. (Protista, Ciliophora) as test species in rapid multilevel ecotoxicity tests. Anglais, 49, p. 271- 280, 2010. 
HICKEY, J. J.,D.W. ANDERSON. 1968. Chlorinated hydrocarbons and eggshell changes in raptorial and fish-eating birds. Science 162/3850: 271-273.

JIANG, J. G.; SHEN, Y. F. Use of the aquatic protozoa to formulate a community biotic index for na urban water system. Science of the Total Environment, v. 346, p. 99-111, 2005.

JIANG, Y., XU, H.; AL-RASHEID, K. A. S.; WARREN, A.; HU, X. Planktonic ciliate communities in a semienclosed bay of Yellow Sea, northern China: annual cycle. Journal of the Marine Biological Association of the United Kingdom, v, 91, n. 1, p. 97-105, 2011.

KERMICHE F., BERREBAH H., DJEBAR M. R., Toxicological effects of drugs (Diclofenac, Ibuprofen, mixture) and Hormesis on a non-target organism: Paramecium sp. Journal of Entomology and Zoology Studies 2016; 4(5): 187-191. E-ISSN: 2320-7078.

KRULL, M.; BARROS, F., 2010, Key Issues in Aquatic Ecotoxicology in Brazil: A Critical Review. J. Braz. Soc. Ecotoxicol., v. 7, n. 2, 2012, 57-66. doi: 10.5132/jbse.2012.02.009

MADONI, P. Ciliated protozoan communities and saprobic evalution of water quality in the hilly zone of some tributaries of the Po River (northen Italy). Hydrobiologia, v. 541, p. 55-69, 2005.

MANSANO, A. S.; MOREIRA, R. A.; PIEROZZI M.; OLIVEIRA T. M.A.; VIEIRA, E. M.; ROCHA O.; REGALISELEGHIM M. H., 2015, Effects of diuron and carbofuran pesticides in their pure and commercial forms on Paramecium caudatum: The use of protozoan in ecotoxicology. Environmental Pollution 213 (2016) 160 - 172. http://dx.doi.org/10.1016/i.envpol.2015.11.054

MIRANDA M. M. P., MARTINS N. F., 2013, Avaliação experimental do efeito tóxico de metais em Paramecium caudatum Ehrenberg. Revista Verde (Mossoró - RN), v. 8, n. 3, p. 247 - 262, Jul - Set, 2013. ISSN 1981-8203.

MOISEENKO, T. I., 2008, Aquatic ecotoxicology: theoretical principles and practical application. Wat. Res., 35: 530-541. doi:10.1134/S0097807808050047

NILSSON J.R., Tetrahymena in cytotoxicology: with special references to effects of heavy metals and selected drugs, Europ J Protistol 25, 2-25(1989b).

PAPADIMITRIOU C.A., PETRIDIS D., ZOUBOULIS A.I., SAMARAS P., YIANGOU M., SAKELLAROPOULOS G.P., 2013, Protozoans as indicators of sequential batch processes for phenol treatment; an auto ecological approach. EcotoxicologyandEnvironmentalSafety98(2013)210-218. http://dx.doi.org/10.1016/i.ecoenv.2013.08.018

RELYEA, R.; HOVERMAN, J., 2006, Assessing the ecology in ecotoxicology: a review and synthesis in freshwater systems. Ecology Letters, (2006) 9: 1157-1171. doi: 10.1111/j.1461-0248.2006.00966.

SAUVANT M. P., PEPIN D., PICCINI E., 1999, Tetrahymena Pyriformis: A Tool For Toxicological Studies. A Review. Chemosphere, Vol. 38, No. 7, pp. 1631-1669, 1999. PIh S0045-6535(98)00381-6

SEILER J. P., 2002, Pharmacodynamic activity of drugs and ecotoxicology-can the two be connected? Swissmedic, Swiss Agency for Therapeutic Products, Erlachstrasse 8, CH-3000 Bern, Switzerland. Toxicology Letters 131 (2002) 105-115. 
ISSN 1980-0827

SEKKAT N., LE DU A., JOUANY J. M., GUERBET M., 1991, Study of interactions between copper, cadmium and ferbam using the protozoan Colpidium campylum bioassay. Ecotoxicology and environmental safety 24,294-300 (1992). 0147-6513/92.

WANICK R. C., PAIVA T. S., CARVALHO C. N., SILVA-NETO I. D., 2008, Acute Toxicity of Cadmium to Freshwater Ciliate Paramecium bursaria. BIOCIÊNCIAS, Porto Alegre, v. 16, n. 2, p. 104-109, jul. 2008.

WERNER, I.; HITZFELD, B., 2012, 50 Years of Ecotoxicology since Silent Spring - A Review. GAIA 21/3 (2012): 217- 224. www.oekom.de/gaia

XU H., SONG W., WARREN A., 2004, An investigation of the tolerance to ammonia of the marine ciliate Euplotes vannus (Protozoa, Ciliophora). Hydrobiologia 519: 189-195, 2004. 